\title{
Short Term Effects of Dietary Boron on Mineral Status in Dairy Cows*
}

\author{
Nuri Baspinar ${ }^{1}$, Abdullah Basoglu ${ }^{2}$, Ahmet Semacan ${ }^{3}$, Erdem Gulersoy $^{2}$ \\ ${ }^{1}$ Department of Biochemistry, Faculty of Veterinary Medicine, Selcuk University, Turkey \\ ${ }^{2}$ Department of Internal Medicine, Faculty of Veterinary Medicine, Selcuk University, Turkey \\ ${ }^{3}$ Department of Gynocology, Faculty of Veterinary Medicine, Selcuk University, Turkey
}

\begin{abstract}
Aim of this study was to obtain knowledge on boron supplemented diet to mineral status of body fluids and feces in short term nutrition of dairy cattle. A total of 24 healthy Holstein dairy cows were used. The animals were fed with standard ration, and boron at three different doses was added to experimental' diets as boron compound: borax, for 10 days. Boron and other macro and trace minerals were determined in serum, milk, urine and feces samples taken on 0 and $11^{\text {st }}$ days. In this study, there were no overt signs of toxicosis, and a pivotal knowledge was obtained in dairy cattle fed with boron supplemented diet on boron absorption, excretion, and its interaction with other minerals. Boron could not completely absorb from gastrointestinal tract. Urine was the most important excretion way of boron. More less boron was also eliminated by milk. Boron levels in body fluids (serum and milk, $p<0.000$ ) were increasingly changed based on the dose. Boron, among minerals, provided a striking increase for $\mathrm{Ca}(p<0.003)$ and $\mathrm{Mg}(p<0.028)$ levels in serum by increasing absorption of these minerals. This topic is worth evaluating as an alternative approach in the prevention of hypocalcemia in transition cows.
\end{abstract}

Keywords-Boron, Macro minerals, Micro minerals, Cattle.

\section{INTRODUCTION}

Today, most nutritionists do not consider a trace element essential unless it has a defined biochemical function in higher animals or humans (Nielsen, 2014a). Trace minerals have critical roles in the key interrelated systems of immune function, oxidative metabolism, and energy metabolism in ruminants. To date, the primary trace elements of interest in diets for dairy cattle have included $\mathrm{Zn}, \mathrm{Cu}, \mathrm{Mn}$, and Se although data also support potentially important roles of $\mathrm{Cr}$, $\mathrm{Co}$, and $\mathrm{Fe}$ in diets (Overton and Yasui, 2014). In vitro, animal, and human experiments have shown that boron is a bioactive element in nutritional amounts whereas boron has received little attention in ruminant nutrition (Nielsen, 2014b; Fry et al., 2011). Boron appears to be of relatively low toxicity to animals. As in previous studies (Owen, 1944; Green and Weeth, 1977), inorganic boron is of low toxicity to cattle. There are many studies on energy, mineral and vitamin supplementation in transition cows in order to prevent production diseases. The objective of the study presented was to determine how dietary boron at different doses affects the distribution of macro and trace minerals in body fluids of dairy cattle.

\section{MATERIAL AND METHOD}

The experimental design was approved by the Committee on Use of Animals in Research of the Selcuk University, Faculty of Veterinary Medicine. A total of 24 pregnant, multiparous Holstein cows in late lactation were enrolled; mean age was 3.5 years, mean 305-day milk production was $6,500 \mathrm{~kg}$, and mean body weight was $650 \mathrm{~kg}$ at the start of the experiment. Cows were fed individually and had free access to tap water.

The cows were randomly allocated to 4 groups: 1 control and 3 experimental, each 6 animals which were offered drinking water $(0.032 \mathrm{mg} \mathrm{B} / \mathrm{L})$ and a basal diet $(0.55 \mathrm{mg} \mathrm{B} / \mathrm{kg})$. Ingredient and daily consumption ratios were corn silage $(12 \mathrm{~kg})$, sugar beet pulp $(10 \mathrm{~kg})$, wheat straw $(4.5 \mathrm{~kg})$ and concentrate $(8.5 \mathrm{~kg})$. The concentrate consisted of $35 \%$ barley, $19.85 \%$ wheat, $15 \%$, wheat bran, $25 \%$ cotton seed meal, $3 \%$ limestone, $0.3 \%$ salt, and $0.35 \%$ vitamin-mineral mixture. It contained $21.5 \%$ crude protein and $2,850 \mathrm{kcal} / \mathrm{kg}$ metabolizable energy. Three experimental groups received $5 \mathrm{~g}, 10 \mathrm{~g}$ and $15 \mathrm{~g}$ of borax (567, 1134 and $1701 \mathrm{mg}$ of boron), respectively as an oral bolus. The bolus administration schedule was chosen to fit within the normal management activities of large dairies, and it required cows to be restrained in headlocks only once daily. 
Blood, urine, milk and feces samples were collected in just before and after the 10-day period. Blood samples were taken from the coccygeal vein. Serum was harvested within an hour by centrifugation for 15 minutes at 3,000 rpm. All the samples were stored at $-20^{\circ} \mathrm{C}$ till analysis. They were analyzed for macro and micro minerals $(\mathrm{B}, \mathrm{Ca}, \mathrm{P}, \mathrm{Mg}, \mathrm{Na}, \mathrm{K}, \mathrm{Mo}, \mathrm{Mn}, \mathrm{Cr}, \mathrm{Cu}$, $\mathrm{Fe}, \mathrm{Ni}, \mathrm{S}, \mathrm{Zn}$ ) by ICP-AES (VARIAN VISTA AX CCD) using Reference Material 8414 (National Institute of Standards and Technology) on day 0 and day $11^{\text {th }}$. Only boron levels were measured on three consecutive days after dietary boron exposure.

All laboratory data were presented as the mean \pm SD. Statistical significance between groups' first and last samples was determined with Paired $t$ test. One Way Anova Posthoc Tukey was applied for statistical significance between groups' last samples' values. Values of $\mathrm{P}<0.05$ were considered significant. SPSS 21.0 program was used for statistical analyses.

\section{RESUlts}

All animals appeared healthy during the boron-diet treatment period. Feed and water consumption were unaffected by treatments. There were no overt signs of toxicosis. Increased boron ingestion increased serum, urine, milk and feces boron concentrations (Table 2). The percentage of boron was increased with the 60,120 and $180 \mathrm{mg}$ of B/kg of diet treatments.

Apart from boron $\mathrm{Ca}, \mathrm{P}, \mathrm{Mg}, \mathrm{Na}, \mathrm{K}, \mathrm{Mo}, \mathrm{Mn}, \mathrm{Cr}, \mathrm{Cu}, \mathrm{Fe}, \mathrm{Ni}, \mathrm{S}, \mathrm{Zn}$ concentrations were also measured in all the samples. Compared to the samples in the beginning of the boron exposure, some changes in macro and trace minerals' level at the end of the experiment were as following: for $\mathrm{Ca}(\mathrm{p}<0.003)$ and $\mathrm{Mg}(\mathrm{p}<0.028)$ concentrations significant increases in serum and significant decreases $(\mathrm{Ca}: \mathrm{p}<0.001, \mathrm{Mg}: \mathrm{p}<0.001)$ in feces were observed in association with boron intake. This data indicates that boron increases $\mathrm{Ca}$ and $\mathrm{Mg}$ absorption. The same thing may be expressed for $\mathrm{Na}$ mineral, because Na was increased in serum $(\mathrm{p}<0.007)$ and decreased $(\mathrm{p}<0.000)$ in feces. It was also decreased in urine. $\mathrm{P}, \mathrm{K}$ and $\mathrm{S}$ concentrations tended to be decreased in serum samples. $\mathrm{Ca}$ and $\mathrm{K}$ concentrations, and $\mathrm{Mg}$ and $\mathrm{P}$ concentrations (only in experimental 3) were significantly decreased in milk samples (Tables 3, 4, 5 and 6). Although these changes are outstanding, it seems to be difficult interpreting them properly.

\section{TABLE 1}

BASAL DIET ANALYSIS

\begin{tabular}{|c|c|}
\hline TSBM $^{\mathrm{f}, \%}$ & 61,39 \\
\hline SE-1X, Mcal $/ \mathrm{kg}^{\mathrm{f}}$ & 2,78 \\
\hline ME-3X, Mcal $/ \mathrm{kg}^{\mathrm{f}}$ & 2,12 \\
\hline NEL-3X, Mcal $/ \mathrm{kg}^{\mathrm{f}}$ & 1,30 \\
\hline NEL-4X, Mcal $/ \mathrm{kg}^{\mathrm{f}}$ & 1,22 \\
\hline NEM-3X, Mcal $/ \mathrm{kg}^{\mathrm{f}}$ & 1,30 \\
\hline NEG-3X, Mcal $/ \mathrm{kg}^{\mathrm{f}}$ & 0,71 \\
\hline Dry matter, \% & 60,96 \\
\hline Crude Protein, $\%$ & 12,82 \\
\hline NDICP, $\%$ & 1,36 \\
\hline ADICP, $\%$ & 0,70 \\
\hline Crude fat, $\%$ & 4,63 \\
\hline NDF, $\%$ & 37,31 \\
\hline ADF, $\%$ & 23,42 \\
\hline NFC, \% & 31,93 \\
\hline Lignin, \% & 5,31 \\
\hline Crude ash, $\%$ & 13,30 \\
\hline $\mathrm{N}$ fraction $\mathrm{A}, \% \mathrm{HP}$ & 17,62 \\
\hline $\mathrm{N}$ fraction $\mathrm{B}, \% \mathrm{HP}$ & 76,94 \\
\hline $\mathrm{N}$ fraction $\mathrm{C}, \% \mathrm{HP}$ & 5,44 \\
\hline RUP, CP $\%, \% 2$, (Bypass Protein ${ }^{\mathrm{f}}$ & 33,45 \\
\hline RUP, CP $\%, \% 4$, (Bypass Protein) ${ }^{\mathrm{f}}$ & 37,64 \\
\hline RUP Sind, $\%^{f}$ & 67 \\
\hline${ }^{\mathrm{f}}$ :calculated by formula & \\
\hline
\end{tabular}


TABLE 2

Boron LeVELS (mg/L).BETWEen Groups' In THE BEGINNING AND AT THE END Of THE EXPERIMENT

\begin{tabular}{|c|c|c|c|c|c|c|c|c|c|c|c|c|}
\hline \multirow[b]{2}{*}{$\begin{array}{l}\text { Body } \\
\text { fluids }\end{array}$} & \multicolumn{4}{|c|}{ Experimental 1} & \multicolumn{4}{|c|}{ Experimental 2} & \multicolumn{4}{|c|}{ Experimental 3} \\
\hline & $\begin{array}{l}\text { Beginning } \\
\text { Mean } \pm \text { SD }\end{array}$ & \multicolumn{2}{|c|}{$\begin{array}{c}\text { End } \\
\text { Mean } \pm \text { SD }\end{array}$} & $\mathbf{P}$ & $\begin{array}{l}\text { Beginning } \\
\text { Mean } \pm \text { SD }\end{array}$ & \multicolumn{2}{|c|}{$\begin{array}{c}\text { End } \\
\text { Mean } \pm \text { SD }\end{array}$} & $\mathbf{P}$ & $\begin{array}{l}\text { Beginning } \\
\text { Mean } \pm \text { SD } \\
\end{array}$ & \multicolumn{2}{|c|}{$\begin{array}{c}\text { End } \\
\text { Mean } \pm \text { SD }\end{array}$} & $\mathbf{P}$ \\
\hline Serum & $0,052 \pm 0,033$ & \multicolumn{2}{|c|}{$0,114 \pm 0,017^{b}$} & 0.018 & $0,061 \pm 0,025$ & \multicolumn{2}{|c|}{$0,136 \pm 0,064^{a b}$} & 0.068 & $0,08 \pm 0,038$ & \multicolumn{2}{|c|}{$0,23 \pm 0,080^{a}$} & 0.008 \\
\hline Milk & $0,051 \pm 0,011$ & \multicolumn{2}{|c|}{$0,131 \pm 0,037$} & 0.001 & $0,038 \pm 0,012$ & \multicolumn{2}{|c|}{$0,114 \pm 0,021$} & 0.000 & $0,003 \pm 0,025$ & \multicolumn{2}{|c|}{$0,089 \pm 0,042$} & 0.001 \\
\hline Urine & $0,689 \pm 0,272$ & \multicolumn{2}{|c|}{$2,081 \pm 1,543$} & 0.015 & $0,893 \pm 0,347$ & \multicolumn{2}{|c|}{$2,132 \pm 1,840$} & 0.032 & $0,494 \pm 0,225$ & \multicolumn{2}{|c|}{$2,721 \pm 1,034$} & 0.000 \\
\hline Feces & $0,085 \pm 0,021$ & \multicolumn{2}{|c|}{$0,144 \pm 0,049^{b}$} & 0.014 & $0,086 \pm 0,019$ & \multicolumn{2}{|c|}{$0,155 \pm 0,027^{b}$} & 0.000 & $0,093 \pm 0,010$ & \multicolumn{2}{|c|}{$0,208 \pm 0,018^{a}$} & 0.000 \\
\hline \multicolumn{13}{|c|}{ Percentage rate of boron (\%) between groups' at the end of the experiment (mg/L). } \\
\hline \multirow[b]{2}{*}{$\begin{array}{l}\text { Body } \\
\text { fluids }\end{array}$} & \multicolumn{4}{|c|}{ Experimental 1} & \multicolumn{4}{|c|}{ Experimental 2} & \multicolumn{4}{|c|}{ Experimental 3} \\
\hline & $\begin{array}{l}\text { Beginnin } \\
\text { g Mean }\end{array}$ & $\begin{array}{c}\text { End } \\
\text { Mean }\end{array}$ & \multicolumn{2}{|c|}{$\begin{array}{c}\text { Percentage } \\
\text { rate } \%\end{array}$} & $\begin{array}{l}\text { Beginning } \\
\text { Mean }\end{array}$ & $\begin{array}{c}\text { End } \\
\text { Mean }\end{array}$ & \multicolumn{2}{|c|}{$\begin{array}{c}\text { Percentage } \\
\text { rate } \%\end{array}$} & $\begin{array}{l}\text { Beginnin } \\
\text { g Mean }\end{array}$ & $\begin{array}{c}\text { End } \\
\text { Mean }\end{array}$ & $\begin{array}{l}\text { Perce } \\
\text { rat }\end{array}$ & Itage \\
\hline Serum & 0,052 & 0,105 & & & 0,061 & $0,136^{\mathrm{b}}$ & & & 0,07 & 0,214 & & \\
\hline Milk & 0.051 & 0,131 & & & 0,038 & 0,114 & & & 0,003 & 0,089 & & \\
\hline Urine & 0,689 & 2,681 & & & 0,893 & 3,132 & & & 0,494 & 2,721 & & \\
\hline Feces & 0.085 & 0.144 & & & 0.086 & 0.155 & & & 0.093 & $0.208^{\mathrm{a}}$ & & \\
\hline
\end{tabular}

Data are expressed as the mean $\pm S D$.

TABLE 3

MiNERAL LEVELS (PPM) IN SERUM SAMPLES IN THE BEGINNING AND AT THE END OF THE EXPERIMENT

\begin{tabular}{|c|c|c|c|c|c|c|c|c|c|}
\hline \multirow[b]{2}{*}{ Minerals } & \multicolumn{3}{|c|}{ Experimental 1} & \multicolumn{3}{|c|}{ Experimental 2} & \multicolumn{3}{|c|}{ Experimental 3} \\
\hline & $\begin{array}{l}\text { Beginning } \\
\text { Mean } \pm \text { SD }\end{array}$ & $\begin{array}{c}\text { End } \\
\text { Mean } \pm \text { SD }\end{array}$ & $\mathbf{P}$ & $\begin{array}{l}\text { Beginning } \\
\text { Mean } \pm \text { SD }\end{array}$ & $\begin{array}{c}\text { End } \\
\text { Mean } \pm \text { SD }\end{array}$ & $\mathbf{P}$ & $\begin{array}{l}\text { Beginning } \\
\text { Mean } \pm \text { SD }\end{array}$ & $\begin{array}{c}\text { End } \\
\text { Mean } \pm \text { SD }\end{array}$ & $\mathbf{P}$ \\
\hline Co & $0.000 \pm 0.000$ & $0.005 \pm 0.002$ & 0.008 & $0.000 \pm 0.000$ & $0.005 \pm 0.004$ & 0.014 & $0.000 \pm 0.000$ & $0.004 \pm 0.003$ & 0.024 \\
\hline Mo & $0.001 \pm 0.001$ & $0.002 \pm 0.001$ & 0.233 & $0.001 \pm 0.001$ & $0.002 \pm .002$ & 0.683 & $0.002 \pm 0.001$ & $0.002 \pm 0.002$ & 0.960 \\
\hline $\mathbf{C a}$ & $5.751 \pm 1.372$ & $13.810 \pm 1.700^{\mathrm{a}}$ & 0.013 & $6.991 \pm 2.626$ & $13.525 \pm 1.556^{\mathrm{a}}$ & 0.003 & $8.205 \pm 3.904$ & $18.859 \pm 2.787^{\mathrm{a}}$ & 0.003 \\
\hline $\mathrm{Cr}$ & $0.000 \pm 0.000$ & $0.003 \pm 0.001^{\mathrm{a}}$ & 0.000 & $0.000 \pm 0.000$ & $0.003 \pm 0.002^{\mathrm{a}}$ & 0.002 & $0.000 \pm 0.000$ & $0.003 \pm 0.001^{\mathrm{a}}$ & 0.002 \\
\hline $\mathbf{C u}$ & $0.033 \pm 0.023$ & $0.031 \pm 0.011$ & 0.832 & $0.036 \pm 0.016$ & $0.025 \pm 0.007$ & 0.164 & $0.040 \pm 0.019$ & $0.042 \pm 0.013$ & 0.591 \\
\hline $\mathbf{F e}$ & $0.225 \pm 0.181$ & $0.116 \pm 0.034$ & 0.228 & $0.147 \pm 0.048$ & $0.098 \pm 0.044$ & 0.139 & $0.139 \pm 0.011$ & $0.122 \pm 0.038$ & 0.264 \\
\hline $\mathbf{K}$ & $12.91 \pm 11.53$ & $12.43 \pm 3.18$ & 0.930 & $19.68 \pm 8.55$ & $11.62 \pm 4.93^{\mathrm{a}}$ & 0.131 & $22.60 \pm 10.48$ & $19.26 \pm 6.87$ & 0.412 \\
\hline Mg & $1.116 \pm 1.168$ & $1.867 \pm .433^{\mathrm{a}}$ & 0.245 & $1.581 \pm .812$ & $1.742 \pm 0.557$ & 0.739 & $1.815 \pm 1.099$ & $2.992 \pm 1.105^{\mathrm{b}}$ & 0.028 \\
\hline Mn & $0.002 \pm 0.002$ & $0.000 \pm 0.000$ & 0.072 & $0.001 \pm 0.000$ & $0.000 \pm 0.000$ & 0.000 & $0.000 \pm 0.000$ & $0.000 \pm 0.000$ & 0.005 \\
\hline $\mathbf{N a}$ & $71.43 \pm 23.01$ & $109.84 \pm 8.29^{\mathrm{a}}$ & 0.022 & $82.34 \pm 19.91$ & $107.40 \pm 12.06^{\mathrm{a}}$ & 0.053 & $84.79 \pm 12.49$ & $124.57 \pm 14.70^{\mathrm{a}}$ & 0.007 \\
\hline $\mathbf{N i}$ & $0.000 \pm 0.000$ & $0.005 \pm 0.002$ & 0.013 & $0.000 \pm 0.000$ & $0.005 \pm 0.003$ & 0.002 & $0.000 \pm 0.000$ & $0.005 \pm 0.002$ & 0.006 \\
\hline $\mathbf{P}$ & $6.998 \pm 6.212$ & $6.530 \pm 1.708$ & 0.865 & $8.918 \pm 3.684$ & $6.205 \pm 2.661$ & 0.249 & $11.374 \pm 6.014$ & $10.978 \pm 4.596$ & 0.855 \\
\hline $\mathbf{S}$ & $55.454 \pm 40.016$ & $48.310 \pm 13.113$ & 0.697 & $68.891 \pm 24.963$ & $47.461 \pm 15.207$ & 0.174 & $84.572 \pm 37.287$ & $73.812 \pm 23.209$ & 0.419 \\
\hline $\mathbf{Z n}$ & $0.042 \pm 0.038$ & $0.056 \pm 0.021$ & 0.436 & $0.063 \pm 0.037$ & $0.050 \pm 0.024$ & 0.488 & $0.0631 \pm 0.034$ & $0.114 \pm 0.040$ & 0.115 \\
\hline
\end{tabular}

Data are expressed as the mean \pm SD

TABLE 4

MINERAL LEVELS (PPM) IN URINE SAMPLES IN THE BEGINNING AND AT THE END OF THE EXPERIMENT.

\begin{tabular}{|c|c|c|c|c|c|c|c|c|c|}
\hline \multirow[b]{2}{*}{ Minerals } & \multicolumn{3}{|c|}{ Experimental 1} & \multicolumn{3}{|c|}{ Experimental 2} & \multicolumn{3}{|c|}{ Experimental 3} \\
\hline & $\begin{array}{l}\text { Beginning } \\
\text { Mean+SD }\end{array}$ & $\begin{array}{c}\text { End } \\
\text { Mean } \pm \text { SD }\end{array}$ & $\mathbf{P}$ & $\begin{array}{l}\text { Beginning } \\
\text { Mean } \pm \text { SD }\end{array}$ & $\begin{array}{c}\text { End } \\
\text { Mean } \pm \text { SD }\end{array}$ & $\mathbf{P}$ & $\begin{array}{l}\text { Beginning } \\
\text { Mean } \pm \text { SD }\end{array}$ & $\begin{array}{c}\text { End } \\
\text { Mean } \pm \text { SD }\end{array}$ & $\mathbf{P}$ \\
\hline Co & $0.000 \pm 0.000$ & $0.005 \pm 0.002$ & 0.001 & $0.000 \pm 0.000$ & $0.007 \pm 0.003$ & 0.003 & $0.000 \pm 0.000$ & $0.005 \pm 0.003$ & 0.001 \\
\hline Mo & $0.002 \pm 0.001$ & $0.004 \pm 0.004^{\mathrm{ab}}$ & 0.264 & $0.001 \pm 0.001$ & $0.009 \pm 0.006^{\mathrm{a}}$ & 0.022 & $0.002 \pm 0.001$ & $0.003 \pm 0.002^{\mathrm{b}}$ & 0.312 \\
\hline $\mathbf{C a}$ & $4.498 \pm 5.136$ & $10.294 \pm .903^{\mathrm{a}}$ & 0.020 & $5.210 \pm 5.649$ & $12.173 \pm 3.021^{\mathrm{a}}$ & 0.022 & $1.716 \pm 0.967$ & $10.939 \pm 1.988^{\mathrm{a}}$ & 0.000 \\
\hline $\mathrm{Cr}$ & $0.000 \pm 0.000$ & $0.003 \pm 0.001^{\mathrm{a}}$ & 0.000 & $0.000 \pm 0.000$ & $0.003 \pm 0.001^{\mathrm{a}}$ & 0.000 & $0.000 \pm 0.000$ & $0.002 \pm 0.001^{\mathrm{b}}$ & 0.002 \\
\hline $\mathbf{C u}$ & $0.002 \pm 0.005$ & $0.000 \pm 0.000$ & 0.291 & $0.001 \pm 0.001$ & $0.000 \pm 0.000$ & 0.048 & $0.002 \pm 0.003$ & $0.000 \pm 0.000$ & 0.039 \\
\hline $\mathbf{F e}$ & $0.025 \pm 0.020$ & $0.070 \pm 0.046$ & 0.076 & $0.0321 \pm 0.008$ & $0.083 \pm 0.055$ & 0.083 & $0.083 \pm 0.130$ & $0.049 \pm 0.022$ & 0.413 \\
\hline $\mathbf{K}$ & $188.38 \pm 88.56$ & $155.51 \pm 62.50^{\mathrm{a}}$ & 0.377 & $247.51 \pm 85.5$ & $184.41 \pm 77.89^{\mathrm{a}}$ & 0.201 & $116.12 \pm 50.42$ & $106.87 \pm 48.52^{b}$ & 0.095 \\
\hline Mg & $21.374 \pm 10.632$ & $27.069 \pm 19.618^{\mathrm{a}}$ & 0.494 & $30.182 \pm 13.290$ & $32.042 \pm 17.395$ & 0.853 & $15.630 \pm 7.598$ & $23.362 \pm 7.327^{\mathrm{a}}$ & 0.055 \\
\hline Mn & $0.000 \pm 0.000$ & $0.000 \pm 0.000$ & 0.000 & $0.001 \pm 0.001$ & $0.000 \pm 0.000$ & 0.012 & $0.005 \pm 0.007$ & $0.000 \pm 0.000$ & 0.090 \\
\hline $\mathbf{N a}$ & $4.584 \pm 5.240$ & $15.56 \pm 15.214^{\mathrm{a}}$ & 0.105 & $14.826 \pm 12.229$ & $17.190 \pm 7.652^{a}$ & 0.167 & $6.647 \pm 6.057$ & $18.680 \pm 33.566^{b}$ & 0.324 \\
\hline $\mathrm{Ni}$ & $0.000 \pm 0.000$ & $0.007 \pm 0.003$ & 0.001 & $0.000 \pm 0.000$ & $0.004 \pm 0.002$ & 0.005 & $0.000 \pm 0.000$ & $0.005 \pm 0.002$ & 0.000 \\
\hline $\mathbf{P}$ & $0.352 \pm 0.122$ & $0.336 \pm 0.210$ & 0.847 & $0.481 \pm 0.119$ & $0.456 \pm 0.264$ & 0.793 & $0.333 \pm 0.134$ & $0.428 \pm 0.300$ & 0.453 \\
\hline $\mathbf{S}$ & $14.399 \pm 6.76$ & $25.200 \pm 15.074^{\mathrm{a}}$ & 0.067 & $19.045 \pm 6.047$ & $36.810 \pm 28.097^{\mathrm{a}}$ & 0.232 & $9.296 \pm 3.913$ & $24.523 \pm 8.859^{a}$ & 0.001 \\
\hline $\mathbf{Z n}$ & $0.000 \pm 0.000$ & $0.007 \pm 0.007$ & 0.029 & $0.007 \pm 0.013$ & $0.016 \pm 0.011$ & 0.179 & $0.047 \pm 0.084$ & $0.039 \pm 0.042$ & 0.796 \\
\hline
\end{tabular}

Data are expressed as the mean $\pm S D$. 
TABLE 5

MINERAL LEVELS (PPM) IN MILK SAMPLES IN THE BEGINNING AND AT THE END OF THE EXPERIMENT.

\begin{tabular}{|c|c|c|c|c|c|c|c|c|c|}
\hline \multirow[b]{2}{*}{$\begin{array}{l}\text { Mine } \\
\text { rals }\end{array}$} & \multicolumn{3}{|c|}{ Experimental 1} & \multicolumn{3}{|c|}{ Experimental 2} & \multicolumn{3}{|c|}{ Experimental 3} \\
\hline & $\begin{array}{l}\text { Beginning } \\
\text { Mean } \pm \text { SD }\end{array}$ & $\begin{array}{c}\text { End } \\
\text { Mean } \pm \text { SD }\end{array}$ & $\mathbf{P}$ & $\begin{array}{l}\text { Beginning } \\
\text { Mean } \pm \text { SD }\end{array}$ & $\begin{array}{c}\text { End } \\
\text { Mean } \pm \text { SD }\end{array}$ & $\mathbf{P}$ & $\begin{array}{l}\text { Beginning } \\
\text { Mean } \pm \text { SD }\end{array}$ & $\begin{array}{c}\text { End } \\
\text { Mean } \pm \text { SD }\end{array}$ & $\mathbf{P}$ \\
\hline Co & $0.000 \pm 0.000$ & $0.005 \pm 0.002$ & 0.000 & $0.000 \pm 0.000$ & $0.005 \pm 0.002$ & .000 & $0.000 \pm 0.000$ & $0.006 \pm 0.001$ & 0.000 \\
\hline Mo & $0.003 \pm 0.001$ & $0.002 \pm 0.001$ & 0.014 & $0.002 \pm 0.001$ & $003 \pm 0.001$ & 438 & $0.003 \pm 0.001$ & $0.002 \pm 0.002$ & 512 \\
\hline $\mathbf{C a}$ & $27.323 \pm 4.443$ & $.156 \pm 8.266$ & 0.739 & $37.580 \pm 14.53$ & $29.804 \pm 9.546^{\mathrm{a}}$ & .229 & $55.575 \pm 16.500$ & $25.502 \pm 5.692^{\mathrm{b}}$ & 0.001 \\
\hline $\mathrm{Cr}$ & $0.003 \pm 0.001$ & $0.006 \pm 0.002^{\mathrm{a}}$ & 0.001 & $0.003 \pm 0.001$ & $0.006 \pm 0.001^{\mathrm{a}}$ & 0.003 & $0.004 \pm 0.002$ & $0.006 \pm 0.002$ & 0.011 \\
\hline $\mathbf{C u}$ & $0.022 \pm 0.003$ & $0.028 \pm 0.007$ & 0.084 & $0.021 \pm 0.004$ & $0.031 \pm 0.009$ & 0.039 & $0.025 \pm 0.009$ & $0.022 \pm 0.003$ & 0.369 \\
\hline $\mathbf{F e}$ & $0.195 \pm 0.022$ & $0.298 \pm 0.066^{\mathrm{ab}}$ & 0.005 & $0.220 \pm 0.102$ & $0.346 \pm 0.081^{a}$ & 0.004 & $0.224 \pm 0.057$ & $0.254 \pm 0.059$ & 0.274 \\
\hline $\mathbf{K}$ & $48.096 \pm 14.91$ & $39.034 \pm 17.514^{\mathrm{a}}$ & 0.309 & $60.171 \pm 18.19$ & $49.086 \pm 14.540^{\mathrm{a}}$ & 0.266 & $92.119 \pm 27.64$ & $36.795 \pm 12.61^{b}$ & 0.001 \\
\hline Mg & $2.468 \pm 0.562$ & $2.667 \pm 1.133$ & 0.67 & $3.579 \pm 1.584$ & $3.462 \pm 1.444$ & 0.885 & $4.960 \pm 1.306$ & $2.648 \pm .908^{\mathrm{a}}$ & 0.001 \\
\hline Mn & $0.002 \pm 0.001$ & $00 \pm 0.000$ & 0.000 & $0.012 \pm 0.020$ & $0.000 \pm 0.000$ & 0.140 & $0.004 \pm 0.002$ & $0.001 \pm 0.002$ & 0.032 \\
\hline $\mathrm{Na}$ & $12.955 \pm 4.241$ & $12.303 \pm 7.286$ & 0.839 & $16.785 \pm 8.310$ & $15.631 \pm 5.349$ & 0.764 & $19.754 \pm 3.146$ & $11.166 \pm 4.382$ & 0.004 \\
\hline $\mathbf{N i}$ & $0.000 \pm 0.001$ & $0.007 \pm 0.003$ & 0.000 & $0.003 \pm 0.002$ & $0.014 \pm 0.014$ & 0.058 & $0.003 \pm 0.002$ & $0.014 \pm 0.015$ & 0.157 \\
\hline $\mathbf{P}$ & $19.110 \pm 4.033$ & $18.179 \pm 8.396$ & 0.773 & $24.149 \pm 8.525$ & $23.327 \pm 10.249$ & 0.872 & $36.206 \pm 9.626$ & $16.834 \pm 6.500^{\mathrm{a}}$ & 0.001 \\
\hline $\mathbf{S}$ & $7.427 \pm 1.558$ & $7.878 \pm 3.208$ & 0.753 & $9.207 \pm 2.959$ & $9.649 \pm 2.555$ & 0.751 & $12.184 \pm 2.836$ & $7.437 \pm 2.483^{\mathrm{a}}$ & 0.004 \\
\hline $\mathbf{Z n}$ & $0.114 \pm 0.015$ & $0.129 \pm 0.033$ & 0.336 & $0.148 \pm 0.055$ & $0.146 \pm 0.044$ & 0.948 & $0.198 \pm 0.062$ & $0.184 \pm 0.137$ & 0.715 \\
\hline
\end{tabular}

Data are expressed as the mean \pm SD

\section{TABLE 6}

MINERAL LEVELS (PPM) IN FECES SAMPLES IN THE BEGINNING AND AT THE END OF THE EXPERIMENT

\begin{tabular}{|c|c|c|c|c|c|c|c|c|c|}
\hline \multirow{2}{*}{ Minerals } & \multicolumn{3}{|c|}{ Experimental 1 } & \multicolumn{3}{c|}{ Experimental 2 } & \multicolumn{3}{c|}{ Experimental 3 } \\
\cline { 2 - 10 } & $\begin{array}{c}\text { Beginning } \\
\text { Mean } \pm \text { SD }\end{array}$ & $\begin{array}{c}\text { End } \\
\text { Mean } \pm \text { SD }\end{array}$ & $\mathbf{p}$ & $\begin{array}{c}\text { Beginning } \\
\text { Mean } \pm \text { SD }\end{array}$ & $\begin{array}{c}\text { End } \\
\text { Mean } \pm \text { SD }\end{array}$ & p & $\begin{array}{c}\text { Beginning } \\
\text { Mean } \pm \text { SD }\end{array}$ & $\begin{array}{c}\text { End } \\
\text { Mean } \pm \text { SD }\end{array}$ & p \\
\hline Co & $0.000 \pm 0.000$ & $0.005 \pm 0.002^{\mathrm{a}}$ & 0.000 & $0.000 \pm 0.000$ & $0.003 \pm 0.002^{\mathrm{b}}$ & 0.007 & $0.000 \pm 0.000$ & $0.005 \pm 0.002^{\mathrm{ab}}$ & 0.000 \\
\hline $\mathbf{M o}$ & $0.005 \pm 0.001$ & $0.005 \pm 0.001$ & 0.656 & $0.005 \pm .002$ & $0.006 \pm 0.002$ & 0.508 & $0.006 \pm 0.001$ & $0.007 \pm 0.003$ & 0.266 \\
\hline $\mathbf{C a}$ & $103.35 \pm 18.35$ & $54.456 \pm 17.27^{\mathrm{a}}$ & 0.001 & $107.18 \pm 25.5$ & $48.285 \pm 10.092^{\mathrm{a}}$ & 0.000 & $114.74 \pm 12.77$ & $71.58 \pm 27.65^{\mathrm{a}}$ & 0.010 \\
\hline $\mathbf{C r}$ & $0.010 \pm 0.007$ & $0.012 \pm 0.002$ & 0.445 & $0.008 \pm .0037$ & $0.013 \pm 0.003$ & 0.000 & $0.007 \pm 0.002$ & $0.017 \pm 0.007$ & 0.008 \\
\hline $\mathbf{C u}$ & $0.069 \pm 0.010$ & $0.074 \pm 0.012$ & 0.465 & $0.069 \pm .0095$ & $0.069 \pm 0.016$ & 0.901 & $0.077 \pm 0.011$ & $0.080 \pm 0.022$ & 0.705 \\
\hline $\mathbf{F e}$ & $2.156 \pm 0.323$ & $2.316 \pm 0.689$ & 0.544 & $2.219 \pm .433$ & $2.143 \pm 0.558$ & 0.573 & $2.349 \pm 0.394$ & $3.142 \pm 1.008$ & 0.097 \\
\hline $\mathbf{K}$ & $4.465 \pm 2.311$ & $14.155 \pm 7.141$ & 0.001 & $6.584 \pm 1.938$ & $12.361 \pm 4.925$ & 0.008 & $7.566 \pm 2.101$ & $9.833 \pm 4.904$ & 0.243 \\
\hline $\mathbf{M g}$ & $46.125 \pm 3.837$ & $23.859 \pm 7.430^{\mathrm{ab}}$ & 0.000 & $46.852 \pm 5.839$ & $21.442 \pm 5.152^{\mathrm{b}}$ & 0.000 & $48.683 \pm 3.249$ & $30.768 \pm 4.990^{\mathrm{a}}$ & 0.000 \\
\hline $\mathbf{M n}$ & $0.233 \pm 0.044$ & $0.279 \pm 0.102$ & 0.301 & $0.241 \pm .0 .536$ & $0.237 \pm 0.046$ & 0.845 & $0.270 \pm 0.025$ & $0.385 \pm 0.154$ & 0.082 \\
\hline $\mathbf{N a}$ & $2.454 \pm 2.077$ & $4.666 \pm 2.919$ & 0.015 & $7.101 \pm 1.436$ & $4.615 \pm 3.166$ & 0.069 & $8.827 \pm 2.831$ & $3.544 \pm 1.551$ & 0.000 \\
\hline $\mathbf{N i}$ & $0.017 \pm 0.005$ & $0.017 \pm 0.007$ & 0.990 & $0.016 \pm 0.004$ & $0.021 \pm 0.008$ & 0.074 & $0.018 \pm 0.001$ & $0.022 \pm 0.007$ & 0.123 \\
\hline $\mathbf{P}$ & $7.430 \pm 1.625$ & $8.332 \pm 3.834$ & 0.586 & $8.344 \pm 1.892$ & $7.176 \pm 2.217$ & 0.058 & $8.145 \pm 1.102$ & $9.133 \pm 4.308$ & 0.572 \\
\hline $\mathbf{S}$ & $5.784 \pm 0.614$ & $6.877 \pm 1.529$ & 0.115 & $6.749 \pm 1.913$ & $6.410 \pm 1.258$ & 0.307 & $7.313 \pm 1.660$ & $7.667 \pm 1.905$ & 0.693 \\
\hline $\mathbf{Z n}$ & $0.223 \pm 0.037$ & $0.286 \pm 0.096$ & 0.127 & $0.244 \pm 0.056$ & $0.276 \pm 0.063$ & 0.079 & $0.254 \pm 0.029$ & $0.356 \pm 0.120$ & 0.064 \\
\hline
\end{tabular}

\section{Data are expressed as the mean $\pm S D$}

\section{DISCUSSION}

This is the first study evaluating dietary boron supplementation at different doses on body fluid distribution of boron, and other macro and trace minerals.

The biochemical function of boron is still speculative. Lactating cows fed with approximately $620 \mathrm{mg}$ of B/100 $\mathrm{kg}$ of body weight/day without toxic effects. Daily intakes of about $765 \mathrm{mg}$ of B/100 kg of body weight (as borax) have deleterious effects (Owen, 1944; Green and Weeth, 1977). The signs of excessive boron ingestion are rather nonspecific (Brook and Boggs 1951). Exposure to large amounts of boron (about $30 \mathrm{~g}$ of boric acid) over short periods of time can affect the stomach, intestines, liver, kidney, and brain and can eventually lead to death in humans. Studies of dogs, rats, and mice indicate that the male reproductive organs, especially the testes, are affected if large amounts of boron are ingested for short or long periods of time. The doses that produced these effects in animals are more than 1,800 times higher than the average daily intake of boron in food by adults in the U.S. population (ARSDR, 2010). One outstanding feature in persons is an erythematous skin rash (Polson and Tattersal, 1969). This rash appears to the redness and edema seen in the legs and around the dew claws of cattle (Green and Weeth, 1977). In this presented study here, adverse effects were not seen with daily 
intakes of 450, 900 and $1350 \mathrm{mg}$ of boron in these 10 days of boron exposure. In other similar study (Weeth et al., 1981) where beef heifers were given drinking water to which $0,15,30,60$ or $120 \mathrm{mg}$ of $\mathrm{B} / \mathrm{L}$ of water was added for 10 days period, it was found that plasma and urine boron levels were significant increased. Similarly to this, in the present study boron levels increased based on the doses in body fluids. Greatest increase of boron was in urine samples, and lowest in faces samples. Serum and milk boron levels were similar. This indicated dietary boron has been much more eliminated by urine than milk and feces.

Macro and trace minerals are essential components of a feeding program for dairy herds and (Swecker, 2014) have important roles in immune function and may affect health in transition dairy cows (Speer and Weiss, 2008). Deficiencies of calcium, phosphorus, and magnesium result in improper skeletal formation. Deficiencies in other macro minerals (e.g., sodium, chloride, potassium) and trace minerals (e.g., copper, zinc, manganese, cobalt, iron) cause biochemical dysfunctions that lead to inefficient metabolism and growth (Smith, 2015).

Boron seems to affect the metabolism of $\mathrm{P}$ as well as $\mathrm{Ca}$ in animal and human models (Hunt, 1988). A significant change in serum $\mathrm{Zn}$ concentration was reported by Kurtoğlu et al. (2005) when chicks were given $5 \mathrm{mg} / \mathrm{kg}$ boron. Chromium levels' increases in serum and urine samples, and sulfur levels' increases in only urine samples at the end of the experiment became outstanding in the present study. Studies indicated that $\mathrm{Cr}$ supplementation may affect health and immune response in ruminants (Spears, 2000). In our previous study (Basoglu et al., 2010), boron (in solution of 1\%) 10, 30 and $50 \mathrm{mg} / \mathrm{kg}$ body weight/day, given to rabbits by oral gavage at $96 \mathrm{~h}$ interval for 7 months, any changes were not observed in serum $\mathrm{Ca}, \mathrm{P}, \mathrm{Mg}$, $\mathrm{Na}, \mathrm{K}$ and $\mathrm{Cl}$ levels. Dietary combination with boron and phytase did not create a synergism with regard to growth performance and bioavailability of the minerals (Cinat et al., 2015). Boron affects blood P and Mg in humans; serum $\mathrm{P}$ concentrations are lower in boron-supplemented subjects than in subjects receiving placebos, and are lower at the end of the study period than during baseline analysis (Meacham et al., 1994).

Effective transition management requires an integrated approach to nutritional and environmental management to provide cows with freedom from rumen disruption, mineral deficiencies, immunosuppression, disorders of lipid metabolism, and other forms of stress (eg, toxic feeds, social disruption) (Sundram, 2015). Clinical milk fever is one of the most recognized diseases of dairy cattle (Oetzel, 2013). Hypocalcaemia around calving is a risk factor for many of these diseases and is an indirect risk factor for increased culling (Goff, 2014). The incidence of clinical hypocalcaemia (milk fever) in the field generally ranges from $0-10 \%$, but may exceed $25 \%$ of cows calving (DeGaris and Lean, 2008). In the present study, boron could not be completely absorbed, a part of dietary boron was eliminated by feces. While $\mathrm{Ca}$ and $\mathrm{Mg}$ levels decreased in feces, their levels in serum increased, associated with boron intake.

\section{CONCLUSION}

Boron increases $\mathrm{Ca}$ and $\mathrm{Mg}$ absorption. This topic is worth evaluating as an alternative approach in the prevention of hypocalcemia in transition cows.

\section{ACKNOWLEDGEMENTS}

This preliminary work was financially supported by TUBITAK (Scientific and Technological Research Council of Turkey, Project No: 213O181)

\section{REFERENCES}

[1] ATSDR (Agency for Toxic Substances and Disease Registry). 2010. Toxicological profile for boron. U:S. Department of Health and Human Services.

[2] Basoglu, A., Baspinar, N., Ozturk, S.A., and Akalin, P.P., 2010. Effects of boron administration on hepatic steatosis, hematological and biochemical profiles in obese rabbits. Trace Elements and Electrolytes, 27:225-231.

[3] Brooke, C. and Boggs, T., 1951. Boric-acid poisoning; report of a case and review of the literature. AMA Am J Dis Child, 82:465-72.

[4] Cinar, M., Kucukyilmaz, K., Bozkurt, M., Catli, A.U., Bintaş, E., et al., 2015. Effects of dietary boron and phytase supplementation on growth performance and mineral profile of broiler chickens fed on diets adequate or deficient in calcium and phosphorus. Br Poult Sci, 56:576-589.

[5] DeGaris, P.J. and Lean I.J., 2008. Milk fever in dairy cows: a review of pathophysiology and control principles. Vet J, 176:58-69.

[6] Fry, R.S., Brown, T.T. Jr, Lloyd, K.E., Hansen S.L., Legleiter, L.R., et al., 2011. Effect of dietary boron on physiological responses in growing steers inoculated with bovine herpesvirus type-1. Res Vet Sci, 90:78-83.

[7] Goff, J.P. 2014. Calcium and magnesium disorders. Vet Clin North Am Food Anim Pract, 30:359-381.

[8] Green, G.H. and Weeth, H.J., 1977. Responses of heifers ingesting boron in water. J Anim Sci, 45:812-818. 
[9] Hunt, C.D., 1998. One possible role of dietary boron in higher animals and humans. Biological Trace Element Research, 66:205-225.

[10] Kurtoglu, F., Kurtoglu, V., Celık, I., Kececi, T. and Nizamlioglu, M., 2005. Effects of dietary boron supplementation on some biochemical parameters, peripheral blood lymphocyte, splenic plasma cell counts and bone characteristics of broiler chicks fed with adequate or inadequate vitamin D3 containing diet. British Poultry Science, 1:87-96.

[11] Meacham, S.L., Taper, L.J. and Volpe, S.L., 1994. Effects of boron supplementation on bone mineral density and dietary, blood, and urinary calcium, phosphorus, magnesium, and boron in female athletes. Environ Health Perspect, 102:79-82.

[12] Nielsen, F.H., 2014a. Should bioactive trace elements not recognized as essential, but with beneficial health effects, have intake recommendations. J Trace Elem Med Biol, 28:406-408.

[13] Nielsen, F.H., 2014b. Update on human health effects of boron. J Trace Elem Med Biol, 28:383-387.

[14] Oetzel, G.R., 2013. Oral calcium supplementation in periparturient dairy cows. Vet Clin North Am Food Anim Pract, 29: 359381.

[15] Overton, T.R. and Yasui, T., 2014. Practical applications of trace minerals for dairy cattle. J Anim Sci, 92:416-426.

[16] Owen, E.C., 1944. The excretion of borate by the dairy cow. J Dairy Res, 13:243-248.

[17] Polson, C.J. and Tattersall, R.N., 1969. Clinical Toxicology 2nd Ed. Philedelphia, Lippincott Co, USA.

[18] Smith, B.P., 2015. Large Animal Internal Medicine. 5th Ed Mosby Elsevier 11830 Westline Industrial Drive St. Louis, Missouri 631.46.

[19] Spears, J.W. and Weiss, W.P., 2008. Role of antioxidants and trace elements in health and immunity of transition dairy cows. Vet J, 176:70-6.

[20] Spears, J.W., 2000. Micronutrients and immune function in cattle. Proc Nutr Soc, 59:587-594.

[21] Sundrum, A., 2015. Metabolic disorders in the transition period indicate that the dairy cows' ability to adapt is overstressed. Animals (Basel), 9:978-1020.

[22] Swecker, W.S. Jr, 2014. Trace mineral feeding and assessment. Vet Clin North Am Food Anim Pract, 30:671-88.

[23] Weeth, H.J., Speth, C.F. and Hanks D.R., 1981. Boron content of plasma and urine as indicators of boron intake in cattle. Am J Vet Res, 42:474-477. 